\title{
Development of the health and economic consequences of smoking interactive model
}

\author{
Michelle E Orme, Susan L Hogue, Lisa M Kennedy, Abigail C Paine, Christine Godfrey
}

\begin{abstract}
Objective-To describe the health and economic consequences of smoking model, a user friendly, web based tool, designed to estimate the health and economic outcomes associated with smoking and the benefits of smoking cessation.

Results-An overview of the development of the model equations and user interface is given, and data from the UK are presented as an example of the model outputs. These results show that a typical smoking cessation strategy costs approximately $£ 1200$ per life year saved and $£ 22000$ per death averted.

Conclusions-The model successfully captures the complexity required to model smoking behaviour and associated mortality, morbidity, and health care costs. Furthermore, the interface provides the results in a simple and flexible way so as to be useful to a variety of audiences and to simulate a variety of smoking cessation methods.

(Tobacco Control 2001;10:55-61)
\end{abstract}

Keywords: consequences of smoking; model; health consequences; economic consequences

Tobacco use is associated with a number of chronic or acute diseases and enormous societal costs. Although the number of people who die or suffer illness because of its use is the best measure of the burden of tobacco, the cost to society must also be measured in terms of economic loss. Tobacco use drains economies of billions of dollars in health care costs and lost productivity each year. As an example, smoking related diseases cost the National Health Service in England between $£ 1.4$ and $£ 1.5$ billion annually, ${ }^{1}$ yet tobacco addiction is a treatable medical condition. For a relatively small initial outlay, smoking cessation interventions result in health gains, which in the long term save lives and reduce the cost of health care for smoking related diseases, releasing resources for other health care programmes.

The methodology described in this paper suggests one approach to developing an epidemiological model for smoking related diseases and the implications of smoking cessation. This methodology was driven by the needs of the end users and the need for a realistic and robust model. The health and economic consequences of smoking (HECOS) model captures the complexity required to model smoking behaviour and associated morbidity, mortality, and costs. This is shown within the paper using data from the UK as an example. The model interface presents these results in a simple, easy-to-use way, allowing different scenarios and combinations of smoking cessation aids to be considered. Users can select different strategies by changing key values. In this way, the model is flexible enough to be useful to a variety of decision makers.

\section{Objectives}

The objective of this work was to develop an interactive software tool that would be widely accessible and applicable for use by health care payers, government policy makers, and other health care organisations in a variety of countries. A key purpose of the model is to show the health and economic burden that smoking related diseases place on health care systems, in addition to showing the potential disease cases averted and the reduction in the smoking related disease costs resulting from successful smoking cessation programmes. The model was commissioned by Glaxo Wellcome for the Lewin Group and offered for use by the World Health Organization (WHO) "European partnership project to reduce tobacco dependence". The model thus currently includes the four target countries of the WHO European partnership project (UK, France, Germany, and Poland), in addition to other European Union countries, as well as Australia, China, Czech Republic, Hungary, Mexico, New Zealand, Norway, and Ukraine.

The purpose of this paper is to outline the stages in the development of the HECOS model and the methodology used to construct the model equations. Additionally, data from the UK are presented as an example of HECOS outputs.

\section{Overview of model development}

To model the burden of smoking in a given population, the relation between different smoking behaviours and the risk of smoking related disease needs to be considered. Therefore, the first stage of the development of the HECOS model was a review of available literature and consultations with appropriate specialists to obtain a comprehensive understanding of the modelling problem.

When there is no overlap between successive generations, population growth can be modelled in discrete steps. Even if a population varies continuously with time, it can be approximated by a discrete system by considering the total population at fixed time intervals. If the time between successive in revised form

20 June 2000 . Accepted

August 2000 
generations is a fixed time step, say $t$, then the population at a time $t+1$, denoted by $N_{t+1}$, can be related to the population at time $t$, denoted by $N_{t}$, using the equation:

$N_{t+1}=f\left(N_{t}\right)$

(equation 1)

where $f(N)$ represents, in general, a non-linear function of $N_{t}$. Discrete difference equations such as equation 1 are widely studied and have a broad range of applications, particularly in the study of population dynamics. $^{2}{ }^{3}$ These simple equations can exhibit complex behaviour ${ }^{4}$ and are usually impossible to solve analytically. The advantage of presenting the model equations in this way is that the variables for future time steps are stated explicitly, in terms of variables from previous time steps. Therefore, it is straightforward to evaluate such equations by using equation 1 recursively.

Markov models are commonly used in health economics to model diseases or interventions that display cyclic behaviour. Markov models are particularly suited to modelling chronic disease, where patients experience disease progression over time..$^{5-7}$ These models use transition probabilities, which give the likelihood of moving to a particular state in the next cycle, given the present state in the current cycle. A Markov model can be written as a set of discrete difference equations of the form:

$n_{t+1}=A(t) n_{t}$

(equation 2)

where $A(t)$ is the transition probability matrix.

The functional form of equation 1 is more comprehensive than the Markov system given in equation 2 , since it is not restricted to transition probabilities or even first order terms. By considering a broader range of functions in our model, the switch from one state to another is not just time driven, but can be dependent upon other factors. As with Markov systems, these equations are based on the assumption of "no memory", in that the transition from one model state to another depends entirely on the current state and is not influenced by events in previous time steps. For example, the likelihood that a smoker will make an attempt to quit smoking may depend on the number of previous quit attempts. While this is a limitation of the model, data pertaining to the impact of prior events is not widely available and a more complex model, such as a system of non-linear differential equations, would certainly decrease the performance of the model interface. In general, a pseudo-Markov model provides a simple and efficient way of modelling the costs and outcomes resulting from health care interventions, particularly for progressive, chronic diseases. ${ }^{56}$

The key activity in such modelling problems is in determining the most appropriate form of the function $f\left(N_{t}\right)$. This function must be carefully chosen in order to capture known or observed behaviour in the study population. Hence, it is good modelling practice to have a full understanding of the modelling problem before attempting to define the model equations. More details of the model assumptions are given in appendix 1 at the end of this article.

CONSTRUCTION OF THE SMOKING MODEL USER INTERFACE

The interface was chosen after examining the needs of the end user and accessibility to the model interface, among other considerations. It was a requirement that the model interface be easy to use and should be made available over the internet. In addition, the model was to be available as a stand alone application, which did not require any special software to support the programme. Further requirements were that the model could be extended at any stage to include additional calculations or extra countries, that key variables could be altered by the user, and that the model should be robust in order to support multiple users and large data sets.

Therefore, the interface was developed using a Microsoft Visual Basic 6 front-end with a Microsoft Access 97 database back end. For the web version, the system was constructed around a Microsoft Visual Basic WebClass. This consists of an ASP page, which makes calls to various ActiveX DLLs. This handles user interactions within the interface, as well as calculation of the model equations and generating outputs in the form of tables and graphs. Further to this, the model equations were translated into a spreadsheet format (Microsoft Excel 97), which provided a testing environment and facilitated the sensitivity analysis.

The advantage of this approach is that a powerful yet flexible application can be produced in a relatively short development time. The stand alone model can be run on most standard computer systems without the need for any additional software support. The web version of the model is hosted by $\mathrm{WHO}^{8}$ and a standard web browser tool such as Microsoft Internet Explorer 4 will allow access to the model.

\section{Results}

The model interface allows the user to amend some key parameters such as the cost, efficacy, and percentage using each of the different smoking cessation methods, the initial number in the model cohort, the discount rate, etc. For the purpose of this paper, the default results for the UK cohort of smokers are presented to demonstrate the possible outputs of the model. For details of the sources used for the default UK data, see appendix 2 on $e T C$ (www.tobaccocontrol.com). In order to determine the validity and comparability of our results with those obtained by other researchers, further calculations were made (not shown in model interface), which were directly compared with published studies.

Tables 1 and 2 show the number of cases of morbidity at any given year and the cumulative number of deaths associated with smoking related disease. It can be seen that smoking related diseases in the UK account for over five 
Table 1 Number of cases of morbidity associated with smoking related disease in the UK

\begin{tabular}{lrrrr}
\hline & \multicolumn{1}{c}{2 years } & \multicolumn{1}{c}{5 years } & 10 years & 20 years \\
\hline COPD & 1794571 & 1965191 & 2364792 & 3236776 \\
Asthma & 487351 & 508018 & 544122 & 615259 \\
CHD & 455679 & 532568 & 626238 & 651600 \\
Stroke & 90602 & 94325 & 109725 & 127293 \\
Lung cancer & 35436 & 43471 & 64384 & 72533 \\
LBW & 97653 & 189524 & 331006 & 571003 \\
Total & 2961292 & 3333097 & 4040265 & 5274464 \\
\hline
\end{tabular}

COPD, chronic obstructive pulmonary disease; CHD,

coronary heart disease; LBW, low birth weight pregnancy.

Table 2 Cumulative number of cases of mortality associated with smoking related disease in the UK

\begin{tabular}{lrrrr}
\hline & 2 years & 5 years & 10 years & 20 years \\
\hline COPD & 38153 & 104048 & 241912 & 653893 \\
Asthma & 56 & 139 & 281 & 568 \\
CHD & 61317 & 167334 & 390627 & 970760 \\
Stroke & 19162 & 48966 & 106199 & 257607 \\
Lung cancer & 37728 & 93469 & 220671 & 548060 \\
LBW & 0 & 0 & 0 & 0 \\
Total & 156417 & 413956 & 959689 & 2430888
\end{tabular}

million cases of smoking related morbidity at 20 years and 2.4 million deaths over the 20 year period.

The model predicts that 79000 smokers will die from one of five smoking related diseases in the first year alone. Table 3 shows a breakdown of these deaths by disease and compares these results to those from Callum. ${ }^{9}$

After 20 years, it is estimated that $56 \%$ of the smoking population in the UK will be affected by or will have died from a smoking related illness (7.5 million out of 13.3 million). This is in agreement with UK/US studies, which show that about half of all smokers are eventually killed by smoking. ${ }^{10}$ The model also predicts that in 20 years $18 \%$ of the current smoking population will have died from a smoking related disease (2.4 million out of 13.3 million). As a comparison, using the figures from Callum ${ }^{9}$ given above, and assuming that the annual number of deaths stay the same over time, approximately 1.8 million smokers would

Table 3 Comparative mortality and morbidity figures

\begin{tabular}{lcccccc}
\hline & COPD & Asthma & CHD & Stroke & Lung cancer & LBW \\
\hline Model results $^{\text {Figures from Callum }}{ }^{6}$ & 18557 & 28 & 29977 & 9602 & 20583 & 0 \\
\hline
\end{tabular}

Table 4 Cumulative health care costs associated with smoking related disease in the UK

\begin{tabular}{lrrrr}
\hline & 2 years & \multicolumn{1}{c}{5 years } & \multicolumn{1}{c}{ 10 years } & \multicolumn{1}{c}{ 20 years } \\
\hline COPD & $£ 592611005$ & $£ 1417270132$ & $£ 2672049381$ & $£ 4782735270$ \\
Asthma & $£ 289938624$ & $£ 679245194$ & $£ 1223108139$ & $£ 2002533494$ \\
CHD & $£ 967977730$ & $£ 2408706686$ & $£ 4631761071$ & $£ 7852225683$ \\
Stroke & $£ 838787789$ & $£ 1947015096$ & $£ 3575206478$ & $£ 6100033102$ \\
Lung cancer & $£ 228703959$ & $£ 532745049$ & $£ 1101550176$ & $£ 2063551665$ \\
LBW & $£ 1417733715$ & $£ 2560759463$ & $£ 3960509932$ & $£ 5524342670$ \\
Total & $£ 4335752822$ & $£ 9545741620$ & $£ 17164185178$ & $£ 28325421884$ \\
\hline
\end{tabular}

Table 5 Comparative health care costs

\begin{tabular}{lccc}
\hline & $\begin{array}{c}\text { Health care cost per } \\
\text { case from model }\end{array}$ & $\begin{array}{l}\text { Total annual health care cost } \\
\text { from model at 1999 prices }\end{array}$ & $\begin{array}{l}\text { Total annual health care cost from } \\
\text { Parrott et al }\end{array}$ \\
\hline COPD & $£ 172.00$ & $£ 301412486$ & $£$ prices \\
Asthma & $£ 308.28$ & $£ 148200759$ & - \\
CHD & $£ 1127.49$ & $£ 483285349$ & $£ 36400000000$ \\
Stroke & $£ 4749.50$ & $£ 432831903$ & $£ 345000000$ \\
Lung cancer & $£ 3044.40$ & $£ 126930451$ & $£ 86000000$ \\
LBW & $£ 14790.52$ & $£ 974284,051$ & - \\
\hline
\end{tabular}

die in the 20 years. The model prediction takes into account the increase in the risk of morbidity and mortality as the cohort ages.

Table 4 illustrates that after 20 years, the cumulative cost of smoking related morbidity is approximately $£ 28.3$ billion, when discounted at $6 \%$, which is the discount rate recommended by the UK government. ${ }^{11}$ These costs are direct medical costs only and do not take into account lost productivity costs such as absenteeism from work or patient costs. The current model costs represent scaled 1999 prices. Using default data for the UK for a cohort of smokers, table 5 shows the total cost of treating smoking related disease in the first year of the model compared to the results from Parrott and colleagues. ${ }^{1}$

There are a variety of methods used when attempting to quit smoking (for example, pharmacological treatment, group counselling, "willpower"). Each method has a different cost and a different chance of success. In this model, the user can use a combination of methods to form a smoking cessation intervention strategy. Table 6 shows the default smoking cessation strategy employed for the analysis of the UK smoking population. The default values for treatment efficacy were taken from the average of those given in a number of published studies. ${ }^{12-15}$ The default values for usage of the interventions were taken from Parrott and colleagues. ${ }^{1}$

A smoking cessation strategy consisting of a combination of pharmacological treatment, general practitioner advice, etc, as shown in table 6 , has a total cost of $£ 103575$ 877. From table 7 , it can be seen that this smoking cessation strategy costs approximately $£ 1200$ per life year saved and $£ 22000$ per death averted. An article published by Tengs and colleagues reports cost-effectiveness estimates for more than 500 life saving interventions, including smoking cessation therapies, which are shown to be more cost-effective than many other disease interventions. ${ }^{16}$ As a comparison with the HECOS model results, the cost per life year saved of smoking cessation advice as given in Tengs and colleagues ranges from $\$ 990$ to $\$ 9800$ (approximately $£ 600-£ 6000$ ).

Table 6 Smoking cessation intervention default strategy for UK

\begin{tabular}{lrlr}
\hline Smoking cessation method & Cost & $\begin{array}{l}\text { Efficacy } \\
(\%)\end{array}$ & $\begin{array}{l}\text { Usage } \\
\text { (Parrott et al. }{ }^{1} \text { ) }\end{array}$ \\
\hline Pharmacological treatment & $£ 77.92$ & 13 & 25 \\
Gneral practitioner advice & $£ 1.84$ & 3 & 10 \\
Group therapy & $£ 91.87$ & 9 & 2 \\
No intervention/willpower & $£ 0.00$ & 1 & 63 \\
Average & $£ 21.50$ & 4.4 & 100 \\
\hline
\end{tabular}

Table 7 Potential impact of smoking cessation strategy in the UK

\begin{tabular}{rccc}
\hline Year & $\begin{array}{l}\text { Total life } \\
\text { years saved }\end{array}$ & $\begin{array}{l}\text { Total deaths } \\
\text { averted }\end{array}$ & $\begin{array}{l}\text { Reduction in cost of } \\
\text { smoking related disease }\end{array}$ \\
\hline 2 & 503 & 503 & $£ 4583295$ \\
5 & 4930 & 1961 & $£ 15921039$ \\
10 & 21967 & 4350 & $£ 28933685$ \\
20 & 85473 & 7533 & $£ 36945579$ \\
\hline
\end{tabular}


SENSITIVITY ANALYSIS

An extensive sensitivity analysis was performed to ensure that the model was robust and that small changes in key model parameters did not lead to a large variation in the results. A brief description of the analyses follows.

Firstly, the annual percentage of smokers who make an attempt to quit smoking was considered. The default for the UK, as obtained from Bridgwood, ${ }^{17}$ was increased in increments of $10 \%$ from $36 \%$ up to $96 \%$ and the result at 20 years was compared. The average effect of this increase was a reduction of 3675 in the number of cases of morbidity, a decrease in health care spending for smoking related disease of $£ 35$ million, and a reduction in smoking related mortality of 10239 cases. The cost of smoking cessation intervention per death averted increases on average by $£ 138$ per increment over the same range, though the cost of smoking cessation intervention per life year saved is reduced on average by $£ 123$.

To examine the effect that uncertainty in the usage or efficacy of individual cessation treatments has on the overall results, the average efficacy rate was varied from $4.4 \%$ to $34.4 \%$, in increments of $5 \%$. An efficacy greater than $34.4 \%$ was assumed to be unachievable given the treatments currently available. A 5\% change in the average efficacy of the smoking cessation strategy leads to an average change of $0.05 \%$ in cases of morbidity (approximately 2551 cases). Furthermore, this achieves an average $0.4 \%$ change in cases of mortality ( 8644 cases) and $0.1 \%$ change in the cost of health care ( $£ 42.3$ million). By increasing the efficacy from $4.4 \%$ to $34.4 \%$, the cessation intervention cost per death averted and cost per life year saved are decreased by $87 \%$ ( $£ 12006$ and $£ 1058$, respectively).

In the model, the percentage of long term quitters relapsing was given as a fixed rate per year. ${ }^{18}$ However, this may overstate the number of long term quitters who recommence smoking as, intuitively, the relapse rate should decrease the longer a person refrains from smoking. Hence, the relapse rate was reduced, in increments of $1 \%$, from $6.9 \%$ to $0 \%$. A decrease of $1 \%$ reduces the number of cases of morbidity over 20 years by an average of 2658 , which decreases health care spending for smoking related disease by an average of $£ 13.6$ million and reduces smoking related mortality by an average of 5623. Furthermore, the cost of smoking cessation intervention per death averted and cost per life year saved decreases by an average of $£ 974$ and $£ 64$, respectively.

Forecasting costs in the future is subject to some degree of uncertainty, particularly as the economic climate and prospective improvements in health care may result in a decrease or increase in future costs. The health care costs in this model were discounted, though outcomes were not. The default discount rate for the UK was taken to be $6 \%$ as per UK government recommendations. ${ }^{11}$ This value was varied from $0 \%$ through to $10 \%$. A $1 \%$ change in the discount rate results in an average change of $8 \%$ in the total health care costs.

\section{Discussion}

The HECOS model is a useful, friendly, flexible, and easily accessible interface. It is unique in that both the health benefits (for example, life years saved) and changes in smoking related health care costs can be simulated over time for the individuals in a population. Hence, the model estimates both health and economic consequences of smoking and smoking cessation.

By allowing users to change key inputs, the model is flexible enough to be useful to a variety of decision makers and can be used to simulate various smoking cessation methods or even new interventions. This flexibility also extends to the model programme which was designed as a database interacting with a visual interface, which can be easily adapted should any changes be required in the long term. For example, it is anticipated that the model will evolve so that it can be populated with new epidemiology or cost data or extended to include other countries as required. Internet technologies have opened up more opportunities for information dissemination and by making such models available over the web, a much wider audience can be reached.

The Office of Smoking and Health in the US Public Health Service has also developed software to permit rapid calculations of death, years of potential life lost, direct health care costs, indirect mortality costs, and disability costs associated with cigarette smoking. ${ }^{19}{ }^{20}$ The software, entitled SAMMEC (smoking attributable mortality, morbidity, and economics costs), aims to provide the USA with data on the health consequences of smoking to policymakers and public health professionals. The SAMMEC software has been further developed and improved upon in recent years, ${ }^{21-23}$ and has been used to calculate US state estimates of Medicaid expenditures attributable to cigarette smoking. The US SAMMEC software has also been modified and used to estimate smoking attributable mortality in Mexico and Canada. ${ }^{24}$

By comparison, the HECOS model is an interactive model, which looks at the current smoking population in 25 countries and predicts the cost and consequences of smoking over a 20 year period for these populations. Furthermore, HECOS allows users to input and compare different smoking cessation strategies, at it is this feature which will be useful to health care planners and policy makers.

One common argument against smoking cessation is that it will increase health care spending in the long term, as it increases life expectancy and hence increases the lifetime cost of health care. A controversial study by Barendregt and colleagues concluded that if all smokers quit, there would be savings in health care costs, but only in the short term. ${ }^{26}$ However, such an argument can be applied to any life extending intervention. The costs should be offset against any health gain, such that the cost-effectiveness of an intervention should be considered. The authors themselves state that public health policy is concerned with health, and smoking therefore should be 


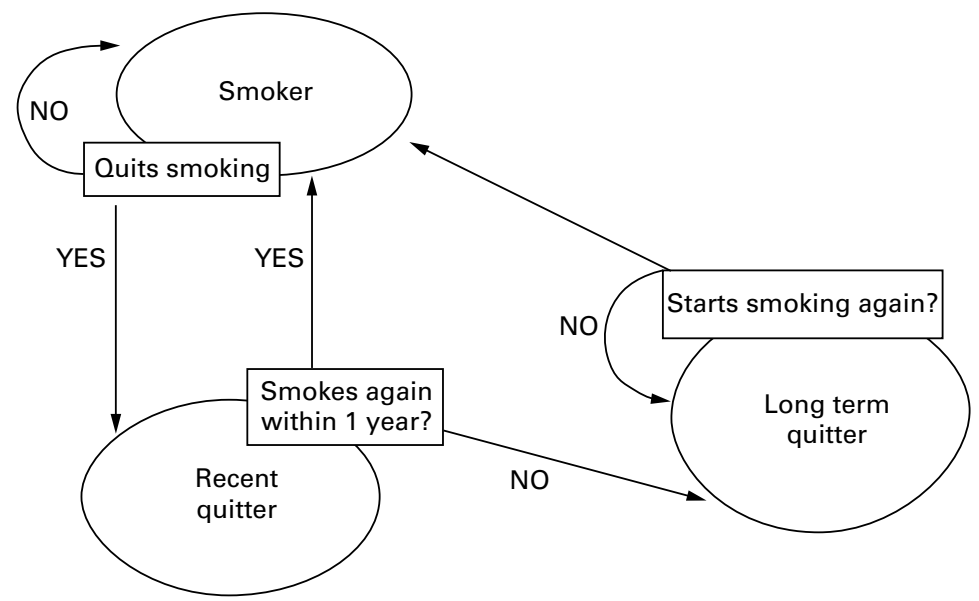

Figure 1 Changes in smoking status.

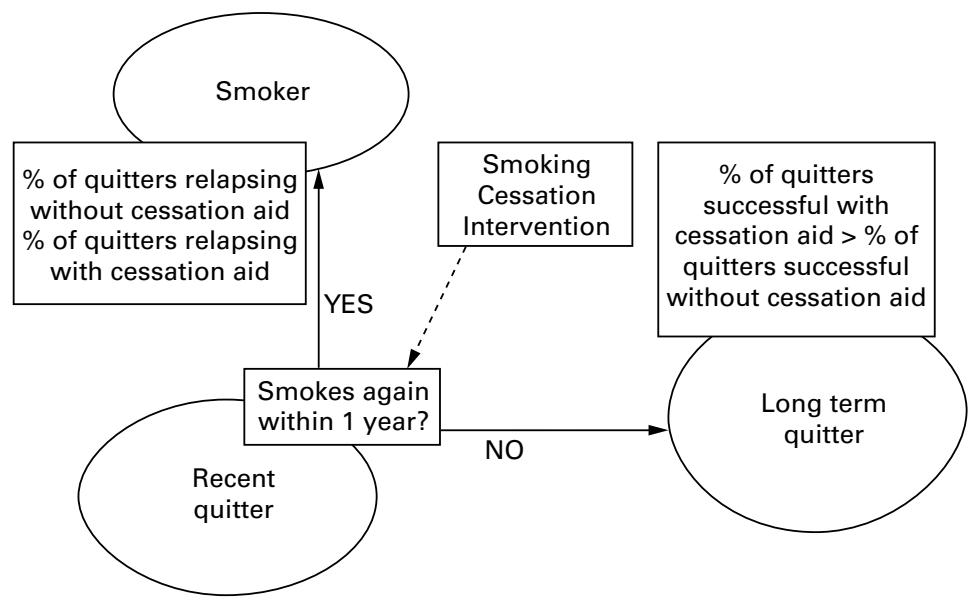

Figure 2 Effect of cessation aid on smoking habit.

discouraged. In any case, it is true to say that for a relatively small initial outlay, smoking cessation interventions result in health gains, which in the long term reduce the cost of health care for smoking related diseases, releasing resources for other health care programmes. ${ }^{1}$ Some authors have questioned the conclusion of the article by Barendregt and colleagues, citing an underestimation of the differences in costs and health care use between smokers and non-smokers. ${ }^{27-31}$

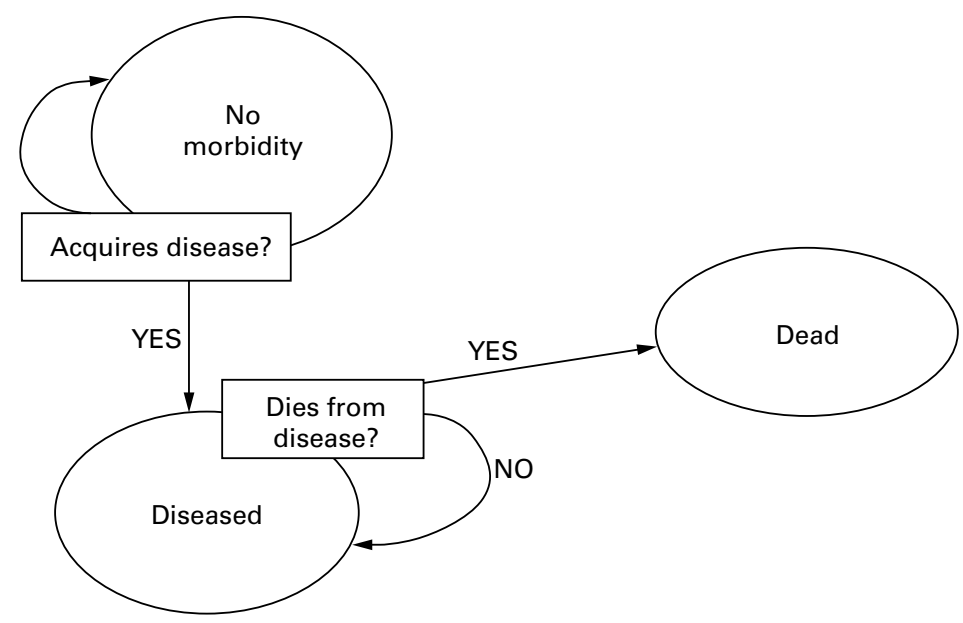

Figure 3 Change of health status.
Furthermore, studies in a number of countries, including the USA, UK, Canada, and China, do not support the notion that the tobacco industry provides some national economic benefit. ${ }^{32}$ It is felt that economic arguments in support of tobacco consumption do not take into account all of the associated costs or the reduction in the quality of life of smokers. This model evaluates the most visible costs associated with tobacco and these indicate that the economic burden of smoking is immense.

The authors are grateful for the contribution of the World Health Organization "European partnership project to reduce tobacco dependence". This study was funded by Glaxo Wellcome Inc. Dr Susan Hogue is a full time employee in the Global Health Outcomes department at Glaxo Wellcome,

\section{Appendix: model assumptions}

CHANGES IN SMOKING BEHAVIOUR

At any time during their smoking career, people can change their smoking habits and may temporarily or permanently quit smoking. It is assumed that a smoker can be in one of three smoking states, namely a current smoker, a recent quitter or a long term quitter. Given that most attempts to quit do not last for more than a few days ${ }^{33}$ we defined a long term quitter to be someone who has not smoked for more than one year. Figure 1 shows the relation between the three smoking states in this model.

EFFECT OF SMOKING CESSATION INTERVENTIONS

Cigarette craving and withdrawal symptoms will have the most impact on those who have recently quit smoking, and it is this group that will benefit most from smoking cessation therapy. Therefore, in the model, it is assumed that smoking cessation aids are targeted at recent quitters and that withdrawal symptoms gradually subside over time. Subsequently, smoking cessation interventions increase the likelihood of a successful quit attempt and long term quitters should have a lower relapse rate compared to recent quitters. These observations are shown in fig 2 .

The impact of a one-off investment in smoking cessation was modelled by increasing the rate at which smokers successfully quit for the first year of the model.

SMOKING AND HEALTH STATUS

Smokers are at risk from smoking related disease and may die prematurely as a result. Ex-smokers can also acquire smoking related diseases, but their risk of disease will be less than that of a current smoker. Figure 3 shows how health status can change for a smoker or an ex-smoker in the model. Note that the change from the no morbidity state to the diseased state is assumed to be irreversible.

The smoking related morbidities included in this model were chronic obstructive pulmonary disease, asthma, chronic heart disease, stroke, lung cancer, and low birth weight pregnancies.

FORMULATION OF THE MODEL EQUATIONS

The model was constructed by considering the change in the health status and smoking behaviour in the smoking population over successive time steps of one year. In general, smoking habits are stable ${ }^{33}$ and a shorter time scale would not capture any change in smoking behaviour. Furthermore, disease and mortality rates are generally measured as a rate per year; therefore, a one year time interval was considered to be the most suitable for this model.

The smoking status of an individual in the model can be split into three discrete (non-overlapping) states, namely:

- a current smoker;

- a recent quitter (defined as an ex-smoker who has quit for less than one year);

- a long term quitter (defined as an ex-smoker who has quit for more than one year). 

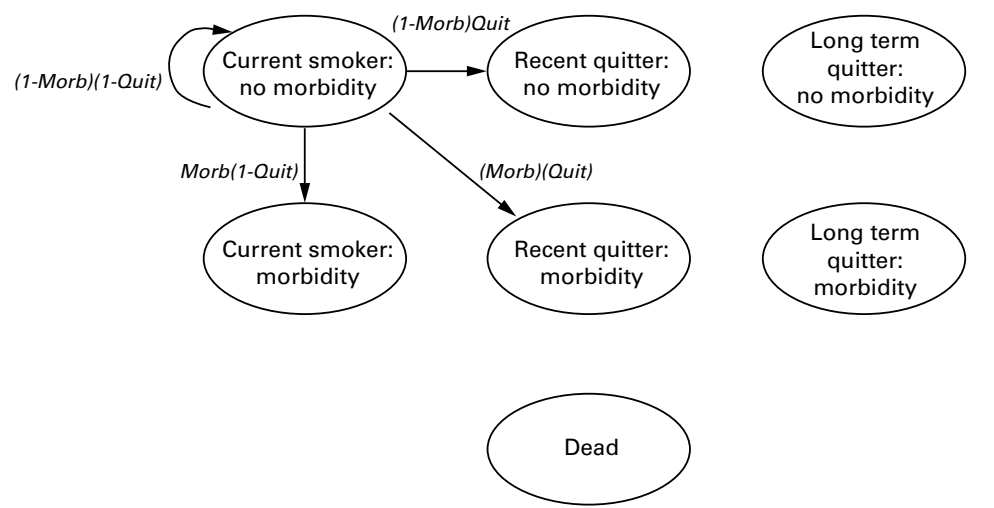

Figure 4 Transitions from the state "current smoker with no morbidity". Quit = rate of quite attempts; Morb = Morb $(a, t)=$ rate of morbidity; $a=$ age/sex group; $t=y$ year.

Furthermore, the health status of an individual can also be split into three mutually exclusive states, namely:

- no morbidity (healthy);

- morbidity (not healthy but alive);

- dead.

Altogether, an individual can be in one of seven model states according to the smoking and health status of that individual. Figure 4 gives a schematic representation of how an individual can move from one state to another. Further schematic representations can be found on $e T C$ (www.tobaccocontrol.com). From this, it can be seen that the number of individuals in a given state $S j$, at time $t+1$, depends on the number in connected states at time $t$, that is:

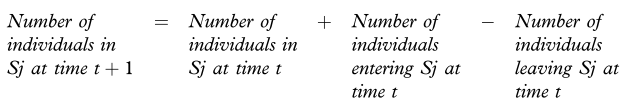

(equation 3)

The discrete difference equations used in the model were constructed using equation 3 , with the transition from one state to another determined by the rate at which smoking behaviour changes (that is, quit rates, relapse rates), as well as disease and mortality rates. A full list of the model equations can be obtained from the author.

Death from non-smoking related causes are not captured in this model. This was because of an absence of comprehensive data pertaining to the relation between the development of smoking or non-smoking related disease.

MODELLING DIRECT HEALTH CARE COSTS

For each year an individual is alive with a smoking related disease, they will accumulate health care costs. The model therefore estimates the cost of health care associated with smoking related disease over a 20 year period.

In general, people will prefer to have something today, rather than at a future time-they have a "time preference". For example, we want the benefits today, but the costs to occur later. As this model predicts costs over 20 years, it is important to take into account the effect of time. In accordance with good economic practice, the future costs in this model were discounted to the present time. $^{34}$

From Drummond and colleagues ${ }^{35}$, if $P$ is the present value, $F n$ the future cost at year $n$, and $r$ is the discount rate, then future costs were discounted to the present value using the formula:

$P=\sum_{n} F n(1+r)^{-n}$

(equation 4)

MODELLING THE EFFECT OF AGE AND SEX

Smoking behaviour and disease risks will vary according to age and sex. In general, more men smoke compared to women and smoking prevalence is higher in younger age groups. To capture this, the smoking population in the model is split into a number of subpopulations according to age and sex. The subpopulations considered in this model were based upon the work of Peto and colleagues ${ }^{36}$, with males aged 0-34, 35-69, and $70+$, and females aged 0-34, 35-69, and 70+.

It is also noted that duration of smoking habit is a better predictor of morbidity than level of smoking. ${ }^{33}$ Therefore, as the smoking population ages, the risk of smoking related disease increases. Hence, in the model the morbidity and mortality rates were time dependent as well as dependent on age and sex.

\section{MODELLING HEALTH GAINS RESULTING FROM SMOKING}

CESSATION

In order to model the effect of smoking cessation, the interface runs two parallel calculations. In the first year of the model, one calculation moves a number of smokers from the recent quitters state to the long term quitters state, according to the efficacy of the smoking cessation strategy chosen. The parallel calculation assumes that the smoking cessation strategy did not take place. The results in subsequent years are then compared to ascertain the benefit of the incremental smoking cessation, which took place in the first year. This approach takes into account those who would have quit smoking anyway, and those who would have subsequently relapsed.

The number of life years saved from a smoking cessation intervention is calculated as follows:

- those who die within model in year $x$, are said to have survived for $x-1$ model years;

- those who survive to the end of the model run are said to have survived 20 years;

- the total number of years survived is: the number who survive $x$ years multiplied by $x$ years, summed over 20 years.

The number of life years saved through an intervention is the difference in the total years survived with an intervention, compared to the total life years survived without an intervention.

1 Parrott S, Godfrey C, Raw M, et al. Guidance for commissioners on the cost effectiveness of smoking cessation interventions. Thorax 1998;53(suppl 5, pt 2):S4-24.

2 Murray JD. Mathematical biology. Biomathematics texts, 2nd ed. Berlin:Springer-Verlag, 1993.

3 Edelstein-Keshet L. Mathematical models in biology. New York: Random House Birkhäuser Mathematics series, 1988

4 May RM, Oster GF. Bifurcations and dynamic complexity in simple ecological models. American Nature 1976;110; 573-99.

5 Briggs A, Sculpher M. An introduction to Markov modelling for economic evaluation. Pharmacoeconomics 1998;13:397-409.

6 Halpern MT, Luce BR, Brown RE, et al. Health and economic outcomes modeling practices: a suggested framework. Value in Health 1998;1:131-47.

7 Sonnenberg FA, Beck JR. Markov models in medical decision making: a practical guide. Medical Decision Making 1993; 13:322-38.

8 World Health Organization. Health and economic consequences of smoking model. <http://www.who.dk/adt/ecos/ whoweb.asp> May 1999.

9 Callum C. The UK smoking epidemic: deaths in 1995. London: Health Education Authority, 1998.

10 Peto R, Chen ZM, Boreham J. Tobacco: the growing epidemic. Nature Medicine 1999;5:15-17.

11 Anon. The Green Book. Appraisal and evaluation in central government. London: Treasury Guidance, 1997.

12 Akehurst R, Piercy J. Cost-effectiveness of the use of Nicorette nasal spray to assist quitting smoking among heavy smokers. British Journal of Medical Economics 1994; 7:155-84

13 Jorenby DE, Leischow SJ, Nides MA, et al. A controlled trial of sustained-release bupropion, a nicotine patch, or both for smoking cessation. N Engl f Med 1999;340:685-91.

14 Law M, Tang JL. An analysis of the effectiveness of interventions intended to help people stop smoking. Arch Intern Med 1995;155:1933-41.

15 Raw M, McNeill A, West R. Smoking cessation: evidence based recommendations for the health care system. $B M 7$ 1999;318:182-5.

16 Tengs TO, Adams ME, Pliskin JS, et al. Five-hundred life-saving interventions and their cost-effectiveness. Risk Analysis $1995 \cdot 15: 369-90$.

17 Bridgwood A, et al. All change? The health education monitoring survey one year on: the 1997 follow-up survey of adults aged 16-74 in England carried out by Social Survey Division of ONS on behalf of the HEA. London: Stationary Office, 1998. 


\section{website}

\section{extra}

Supplemental data and schematic representations appear on the Tobacco Control website

www.tobaccocontrol.com
18 US Department of Health and Human Services. The health benefits of smoking cessation. A report of the Surgeon General, 1990. Rockville, Maryland: Public Health Service, Cc for Disease Control, Office on Smoking and Health, 1990.

19 Shultz JM, Novotny TE, Rice DP. Quantfying the disease impact of cigarette smoking with SAMMEC II software. Public Health Rep 1991;106:326-33.

20 Anon. Cigarette smoking-attributable mortality and years of potential life lost-United States, 1990. MMWR Morb Mortal Wkly Rep 1993;42:645-9.

21 Miller LS, Zhang X, Novotny T, et al. State estimates of Medicaid expenditures attributable to cigarette smoking, fiscal year 1993. Public Health Rep 1998;113:140-51.

22 Miller IS, Zhang X, Rice DP, et al. State estimates of total medical 1993. Public Health Rep 1998;113:447-58.

23 Miller, LS. Smoking estimate correction. Public Health Rep 1998;113:191.

24 Smoking-attributable mortality-Mexico, 1992. MMWR Morb Mortal Wkly Rep 1995;44:372-3, 379-81.

25 Makomaski Illing EM, Kaiserman MJ. Mortality attributable to tobacco use in Canada and its regions, 1994-1996. .

26 Barendregt JJ, Bonneux L, van der Maas PJ. The health care costs of smoking. N Engl f Med 1997;337:1052-7.
27 Hodgson TA. The health care costs of smoking [letter].N Engl F Med 1998;338:470.

28 Fries JF. The health care costs of smoking [letter] N Engl 7 Med 1998;338:470-1.

29 Heaney D. The health care costs of smoking [letter]. N Engl f Med 1998; 338(7):471.

30 Leistikow BN, Miller TR. The health care costs of smoking [letter]. N Engl F Med 1998;338:471.

31 Sauter C. The health care costs of smoking [letter]. N Engl F Med 1998;338:471-2.

32 World Health Organisation. Tobacco-supporting the tobacco industry is bad economics. WHO Information Fact Sheet No. 223. April 1999. http:\|www.who.int/inf-fs/ en/fact223.html. Accessed November 1999.

33 Jarvis MJ. Patterns and predictors of smoking cessation in the general population. In: Bolliger CT, Fagerstrom KO, eds. The tobacco epidemic. Prog Respir Res.1997;28 eds. The tobacco

34 Eisenberg JM. Clinical economics. A guide to the economic analysis of clinical practices. FAMA 1989;262:2879-86.

35 Drummond MF, O'Brien B, Stoddart GL, et al. Methods for the economic evaluation of health care programmes, 2 nd ed. .

36 Peto R, Lopez A D, Boreham J, et al. Mortality from smoking in developed countries 1950-2000. Oxford: Oxford University Press, 2000.

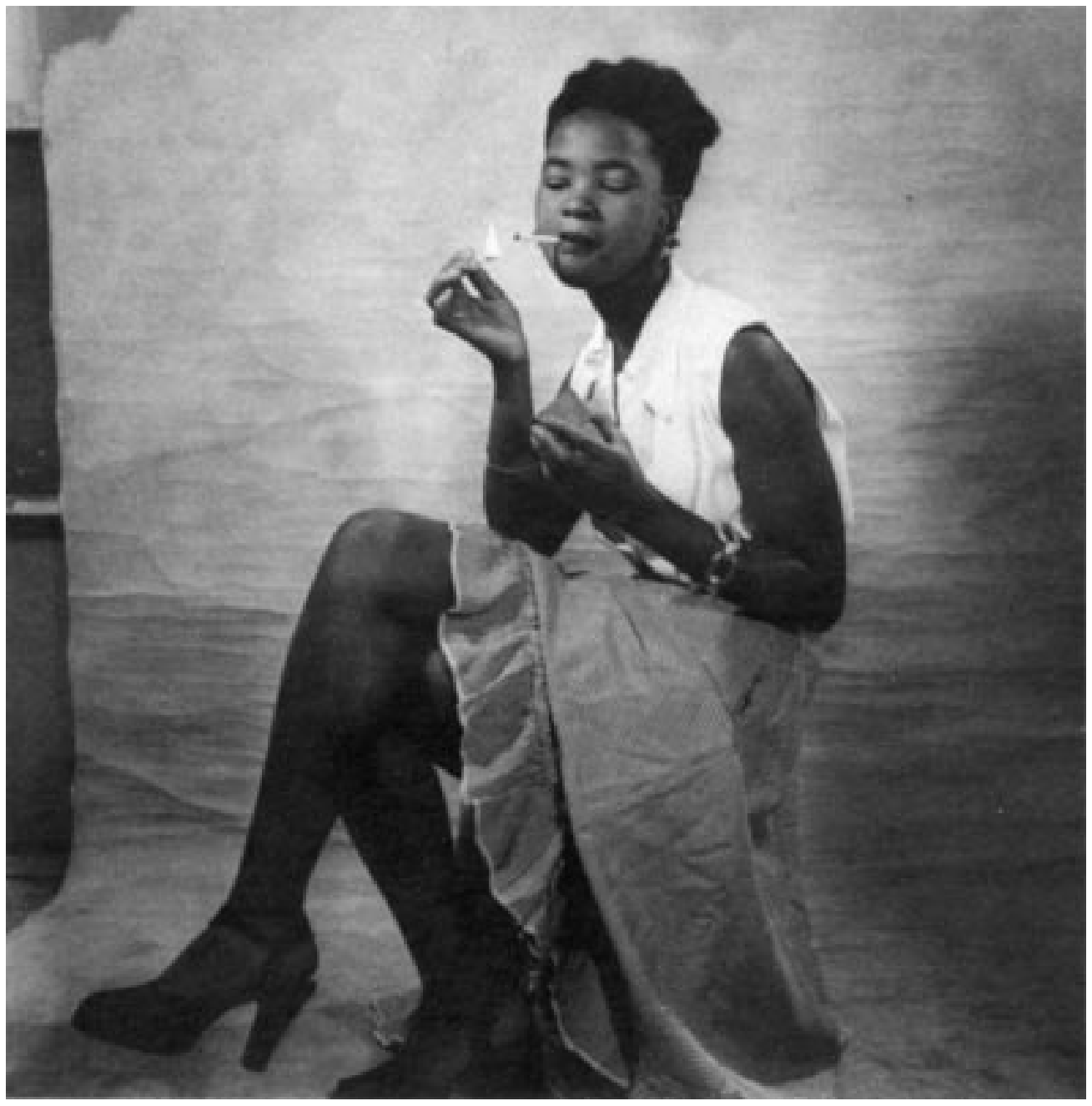

Contributed by Melanie Wakefield. 